Article

\title{
Non-Contact Sensing Testbed for Post-Surgery Monitoring by Exploiting Artificial-Intelligence
}

\author{
Mohammed Ali Mohammed Al-hababi ${ }^{1}$, Muhammad Bilal Khan ${ }^{1}$, Fadi Al-Turjman ${ }^{2}{ }^{(D)}$ \\ Nan Zhao ${ }^{1}$ and Xiaodong Yang ${ }^{1, *}$ \\ 1 School of Electronic Engineering, Xidian University, Xi'an 710071, China; \\ mohammed_al-hababi@stu.xidian.edu.cn (M.A.M.A.-h.); bilal@stu.xidian.edu.cn (M.B.K.); \\ nan_zhao_@hotmail.com (N.Z.) \\ 2 Artificial Intelligence Department, Research Center for AI and IoT, Near East University, Nicosia, \\ Mersin 10, Turkey; Fadi.alturjman@antalya.edu.tr \\ * Correspondence: xdyang@xidian.edu.cn
}

Received: 28 May 2020; Accepted: 14 July 2020; Published: 16 July 2020

\begin{abstract}
Non-contact health care monitoring is a unique feature in the emerging $5 \mathrm{G}$ networks that is achieved by exploiting artificial intelligence (AI). The ratio of the number of health care problems and patients is increasing exponentially and creating burgeoning data. The integration of AI and Internet of things (IoT) systems enables us to increase the huge volume of data to be generated. The approach by which AI is applied to the IoT systems enhances the intelligence of the health care system. In post-surgery monitoring of the patient, timely consultation is essential before further loss. Unfortunately, even after the advice of the doctor to the patient, he/she may forget to perform the activity in the correct way, which may lead to complications in recovery. In this research, the idea is to design a non-contact sensing testbed using AI for the classification of post-surgery activities. Universal software-defined radio peripheral (USRP) is utilized to collect the data of spinal cord operated patients during weight lifting activity. The wireless channel state information (WCSI) is extracted by using orthogonal frequency division multiplexing (OFDM) technique. AI applies machine learning to classify the correct and wrong way of weight lifting activity that was considered for experimental analysis. The accuracy achieved by the proposed testbed by using a fine K-nearest neighbor (FKNN) algorithm is $99.6 \%$.
\end{abstract}

Keywords: Artificial intelligence (AI); fine K-nearest neighbor (FKNN); IoT; orthogonal frequency division multiplexing (OFDM); universal software-defined radio peripheral (USRP); wireless channel state information (WCSI)

\section{Introduction}

Invention regarding human health is becoming more popular in the science world. Intelligent and regular health monitoring is necessary to diagnose vital signs such as human respiration, heart rate, etc., at the early stage. In particular, regular monitoring of the patient's state of health is mandatory to avoid significant losses. Healthcare monitoring requires reliable and efficient follow-up on regular operations, resources, and services. Smart healthcare systems need artificial intelligence (AI) and the Internet of things (IoT) to develop smart cities that reduce patient waiting time [1]. Several challenges still exist in ubiquitous healthcare systems such as frequent mobility, scalability, flexibility, reliability, and energy efficiency issues. Successful deployment of smart e-health gateways can ensure that the ubiquitous health monitoring systems are used extensively, especially in the smart hospital environment [2]. Quantifying uncertainty in cloud-based computing for different environments is an important factor for reliability in various configurations [3]. It is essential to build highly secure features based on 
smart healthcare applications [4]. The development of intelligent and smart medical services reduces the likelihood of death [5]. When solving health care problems, researchers participated in the development of intelligent platforms and the search for promising solutions. According to international perspectives on spinal cord injury from the World Health Organization (WHO) and the International Spinal Cord Society (ISCOS), international incidence data suggest that each year between 250,000 and 500,000 people become spinal cord injured. Most of these cases are traumatic spinal cord injury, the leading causes of which are road traffic injuries, violence, and falls. Usually, these cases require surgical intervention [6]. Even though there are many systems in place for health monitoring, existing platforms such as computer vision, wearable devices, or sensors are relatively mature $[7,8]$, but they have a lot of disadvantages; for example, the computer vision platform cannot be used in private places such as the bathroom and changing room, whereas the wearable sensor platform can be uncomfortable when wearing it all the time, especially for elders and children, and also some of existing platforms do not work in real-time.

Studies have shown that human existence and their movements affect the channel state information (CSI) of Wi-Fi signals using OFDM technology $[9,10]$. CSI has an advantage over light, infrared, or thermal energy when trying to infer people's movements [11,12]. WCSI based detection techniques were successfully applied to several applications, such as respiration detection and fall detection [13,14]. These techniques completely depend on AI based machine learning algorithms that require training data for all the detection of target activities [15]. In this paper, our goal was to develop a smart health care system in which computation and data storage are closer to the place where it is needed, and to improve reliability, flexibility, scalability, and portability.

The primary contributions of this paper are summarized below:

- We verify the feasibility of non-contact sensing using USRP by exploiting AI for post-surgery activities detection, especially weight lifting activity. To the great of our knowledge, this is the first work using universal software-defined radio peripheral (USRP) to extract WCSI for healthcare purposes and to monitor patients' activities.

- We suggest a dynamic feature extraction technique of WCSI, which efficiently decreases the influence of environmental changes.

- We only depend on training data derived in ordinary situations. We collect data when a patient is normally lifting things and feed them into an algorithm to avoid collecting the data after when the patient had surgery, which is hard to collect in practical use.

- We measured the execution of this technique in an actual environment, which we perform in our lab. The experimental outcomes show that the post-surgery activities monitoring testbed has high detection performance in a line of sight (LOS) as well as non-line-of sight (NLOS) scenario.

The rest of this paper is arranged as follows: In Section 2, related work is presented. In Section 3, there is a design overview of the post-surgery activities monitoring testbed that can be applied to various health care applications. In Section 4 , the experimental setup and the laboratory environment are discussed. In Section 5, discussion on performed experiments, testbed performance, and results are evaluated. Section 6 concludes the paper and recommends the improvements in the form of future work for this research. The abbreviations used for more readability in this paper are defined in Table 1. 
Table 1. List of used abbreviations.

\begin{tabular}{cccc}
\hline Abbreviation & Description & Abbreviation & Description \\
\hline ADC & Analog to Digital Converter & LOS & Line of Sight \\
AI & Artificial Intelligence & LPA & Log Periodic Antenna \\
CP & Cyclic Prefix & LPF & Low Pass Filter \\
DAC & Analog Conversion & LSVM & Linear Support Vector Machine \\
DAC & Digital to Analog Converter & NLOS & Non-Line-of Sight \\
DC & Direct Current & OFDM & Orthogonal Frequency Division \\
DDC & Digital down Converter & PCA & Principal Component Analysis \\
DUC & Digital up Conversion & QPSK & Quadrature Phase Shift Keying \\
EBT & Ensemble Boosted Trees & RF & Radio Frequency \\
FDT & Fine Decision Tree & RGB & Red Green Blue \\
FFT & Fast Fourier Transform & RSS & Received Signal Strength \\
FKNN & Fine K-Nearest Neighbor & TA & Transmit Amplification \\
IFFT & Inverse Fast Fourier Transform & ToF & Time of Flight \\
ISCOS & International Spinal Cord & USRP & Universal Software-Defined \\
& Society & Radio Peripheral \\
ISI & Inter Symbol Interference & WCSI & Wireless Channel State \\
LDA & Linear Discriminant Analysis & WHO & Information \\
\end{tabular}

\section{Related Work}

In this section, we concisely look through related work on health care and non-contact sensing.

\subsection{Traditional Health Care Techniques}

Traditional health care techniques are classified into three types: computer vision-based, wearable device-based, and ambient sensor-based techniques $[16,17]$. The computer vision-based techniques use low price RGB cameras and depth cameras to assess the state of a patient [18]. Miguel et al. introduced the RGB camera-based fall detection system, which is a device-free technique [19]. The fall detection system accomplished very good detection performance with a detection rate of greater than $96 \%$. Lotfi et al. proposed a vision-based fall detection technique that deals with occlusion issues by using head tracking [17]. Balakrishnan et al. suggested a camera-based heart rate monitoring technique using subtle heart techniques caused by a pulse [20]. The experimental assessment showed that the detected heart rate was nearly the same as the heart rate measured by an electrocardiogram device. The computer vision-based techniques invade users' privacy, which is critical for monitoring in private places. The computer vision-based techniques also receive environmental limitations; there can be blind spots in the NLOS environment.

The wearable device technique utilizes wearable sensors, for example, the gyroscope, accelerometer, and light intensity sensor, to detect the patient's safety state while preserving the patient's privacy. Jain et al. introduced a portable fall-detection device connected to the neck of a vest [21]. Sabatini et al. introduced a fall detection technique utilizing an inertial sensor and barometer [22]. These techniques compute the root-mean-square accelerometer data and/or other features and detect the large impact. An obvious weakness of the wearable device technique is a deterioration in the comfort of patients when performing movements after surgery. Particularly patients with dementia could also forget to wear the wearable devices.

The ambient sensor-based techniques use sensors such as vibration, pressure, sound, and radar embedded in an environment to recognize the activities of humans. Zigel et al. introduced a fall detection system that depends on vibration and sound generated by a fall event [23]. However, the ambient sensor-based techniques suffer from high false detections because of noise such as everyday object falls [24]. Radar-based device free techniques have also been introduced [25,26]. 
The radar-based techniques need a line-of-sight (LOS) environment, i.e., no occlusion is allowed between the radar and a patient, which extremely restricts physical deployment

\subsection{Using Wi-Fi Communication Signals for Sensing}

In the past few years, the use of Wi-Fi signals for sensing has proven to have great potential within the field of device-free sensing. The major power of Wi-Fi sensing is that it is often applied not only to LOS environments but also to NLOS environments. Wi-Fi sensing technologies are widely studied for indoor localization and movement recognition. As an example of conventional Wi-Fi sensing technologies, Sugano et al. introduced an indoor localization system utilizing received signal strength (RSS) in a Wi-Fi sensor network [27]. The Chronos system was introduced, in which an indoor localization system achieved decimeter-level localization employing a single Wi-Fi access point [28]. The Chronos system uses CSI derived from commodity Wi-Fi devices to calculate distances between multiple antennas and a target device based on the time of flight (ToF) of Wi-Fi signals. The Chronos system is similar to state-of-the-art localization systems that need four or five access points.

Sensing techniques based on CSI were also introduced. Ali et al. designed a keystroke recognition technique utilizing CSI [29]. To locate fingers pushing keys on CSI, keystroke features are extracted by playing principal component analysis (PCA). The keystroke recognition technique achieves a word recognition accuracy of greater than $85 \%$. RT-Fall is a fall down detection system using Wi-Fi signals [13]. RT-Fall uses the phase difference between antennas for fall detection and obtains high accuracy. RF-Pose is a through the wall human pose estimation technique utilizing Wi-Fi signals [30]. RF-Pose draws heat maps in two dimensions via a deep neural network to estimate human posture behind walls. Experimental assessments show that RF-Pose can describe human posture in detail [31]. However, post-surgery multiple posture activity detection is still a challenge, so in this research, we detect and monitor multi-pose complex weight lifting activity.

\section{Materials and Methods}

In post-surgery activities monitoring, the design testbed consists of two USRP devices and two Laptops/PCs as shown in Figure 1. Two omnidirectional antennas are utilized to capture the LOS and NLOS based WCSI of the weight lifting activity. The testbed consists of two sections. The first section is about hardware, where we use USRP devices for the processing of RF signals, and the second is software, where MATLAB/Simulink is used for implementing OFDM signal generation and reception and further classification using machine learning algorithms.

\subsection{Hardware Functionality}

In hardware functionality, the transmitter performs digital up-conversion (DUC), digital to analog conversion (DAC), low-pass filtering (LPF), mixer, and transmit amplification (TA) for the setting of transmitter gain, while the low noise amplification (LNA), mixer, LPF, analog to digital converter (ADC), and digital down converter (DDC) are the operations of the hardware receiver side. The following hardware is used in the experiments: the PC on the transmitter side is a Lenovo, Intel(R) Core (TM) i5-7400 $3.00 \mathrm{GHz}$ processor, 8 GB RAM, and Windows 10' 64-bit operating system. The PC on the receiver side is a Lenovo, Intel(R) Core (TM) i5-7500 $3.40 \mathrm{GHz}$ processor, $16 \mathrm{~GB}$ RAM, and Windows 10' 64-bit operating system. Two USRP devices are used and their hardware setting parameters are given in Table 2.

\subsection{Software Functionality}

The MATLAB/Simulink version R2019a software is used for implementation. This software part consists of two main blocks, i.e., the first one is the transmitter and the second is the receiver block as depicted in Figure 2. Binary random bits are input to the OFDM transmitter block to generate the OFDM symbols and to utilize the USRP transmitter block for sending the signal. The USRP receiver 
block receives the signal and WCSI is extracted using the frequency response by applying the FFT at the receiver. The software parameters are shown in Table 3.
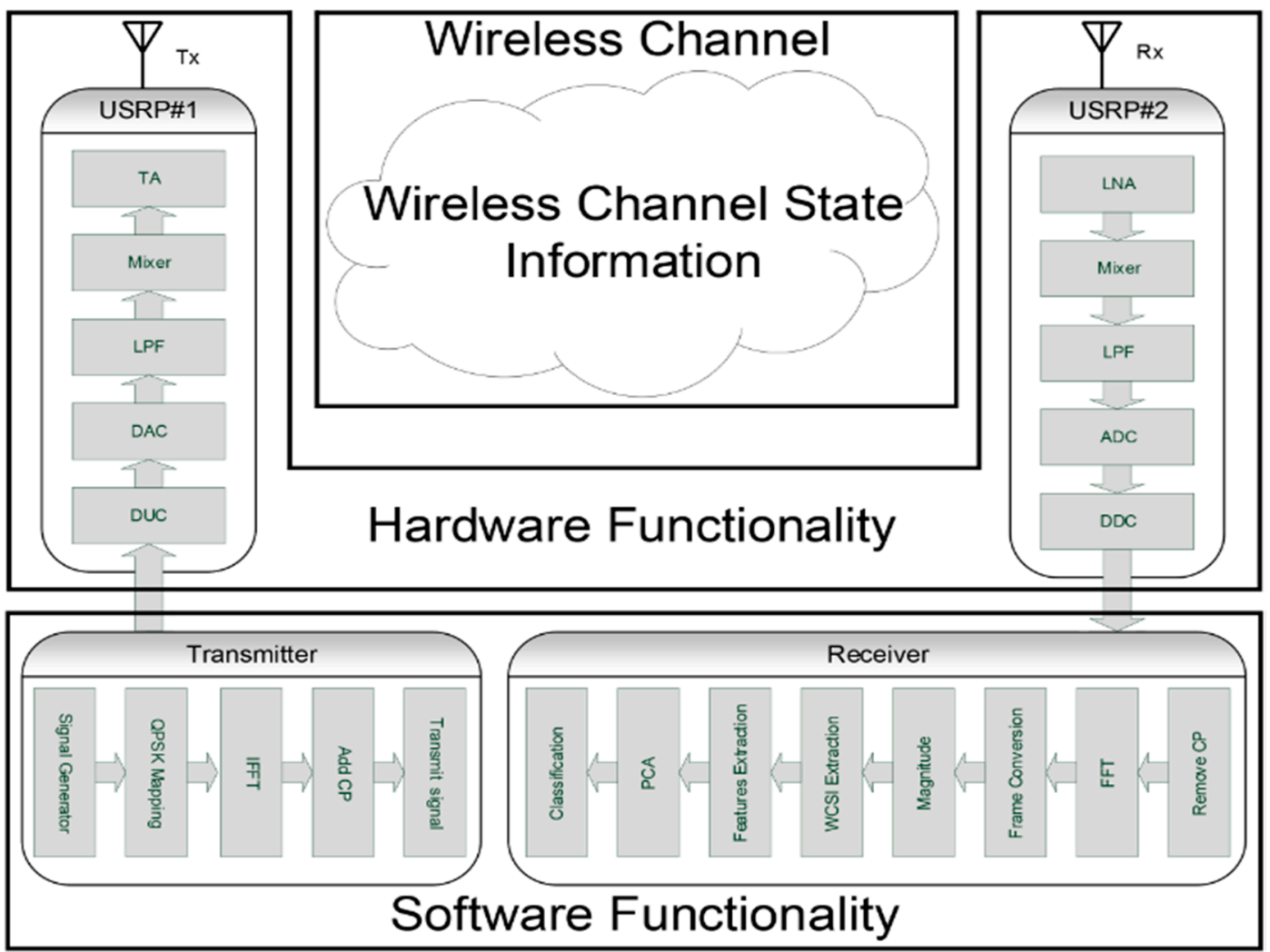

Figure 1. Non-contact sensing testbed architecture.

Table 2. Hardware parameters.

\begin{tabular}{cc}
\hline Testbed & USRP B210 \\
\hline Antenna & Omni-directional \\
Device Frequency Range & $70 \mathrm{MHz}-6 \mathrm{GHz}$ \\
Channel Mapping Rx & 1 \\
Channel Mapping Tx & 1 \\
Center Frequency & $5.32 \mathrm{GHz}$ \\
Clock Source \& PPS Source & Internal \\
Master Clock Rate & $200 \mathrm{MHz}$ \\
Interpolation Factor & 250 \\
Enable Burst mode & False \\
Transport data type & int16 \\
Decimation Factor & 250 \\
Output data type & $30 \mathrm{AD} 2 \mathrm{FE}$ \\
Transmitter serial number & $30 \mathrm{AD} 311$ \\
Receiver serial number & 70 \\
Transmitter Gain & 50 \\
Receiver Gain & 80 \\
Samples per frames &
\end{tabular}



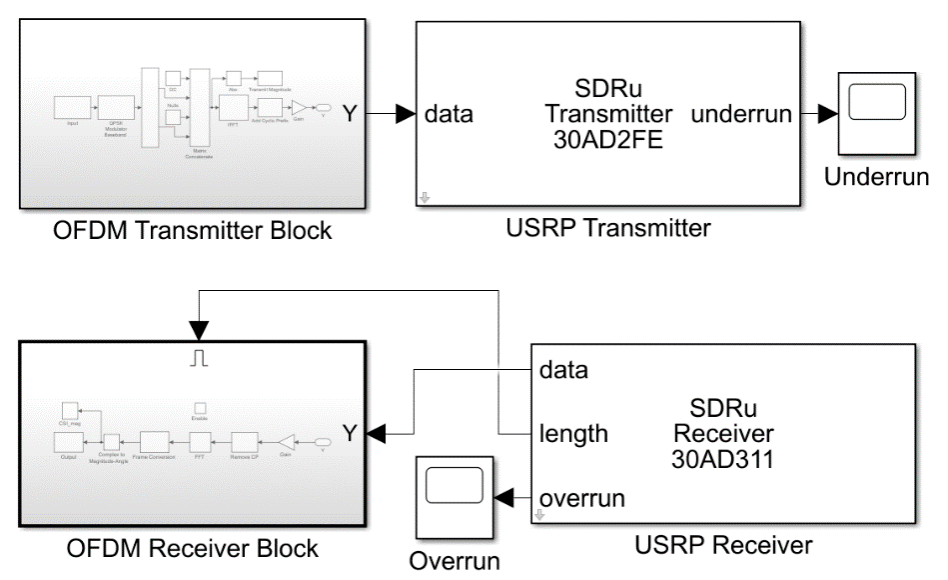

Figure 2. Simulink model.

Table 3. Software parameters.

\begin{tabular}{cc}
\hline Parameter & Values/Type \\
\hline Input Bits & 104 \\
Bits per Symbols (M) & 2 \\
Modulation type & QPSK \\
OFDM subcarriers & 64 \\
Data subcarriers & 52 \\
Null subcarriers & 11 \\
DC & 1 \\
Used subcarriers & 52 \\
NFFT & 64 \\
Cyclic prefix & NFFT - Data subcarriers \\
Sampling Frequency & 80,000 \\
Samples per frames & 80 \\
\hline
\end{tabular}

\subsubsection{Transmitter Process}

As shown in Figure 3, bits are generated and the information maps a vector of bits. These bits are modulated by utilizing the modulation technique quadrature phase-shift keying (QPSK). The applied signal can be either integers or bits. The width must be an integer multiple of the incoming bits per symbol when setting the 'Input type' parameter to 'Bit.' The De-mux block divides vector signals into smaller vectors or scalars, and these are utilized for the series to parallel transformation. After that, nulls and DC subcarriers are added to create a 64-point OFDM signal, to transform complex frequency domain signals into time-domain by utilizing the IFFT block, and to implement orthogonality between the subcarriers. At the end, the cyclic prefix (CP) is inserted to prevent inter-symbol interference (ISI) and then it is sent to up-sample and DAC in the USRP hardware block.

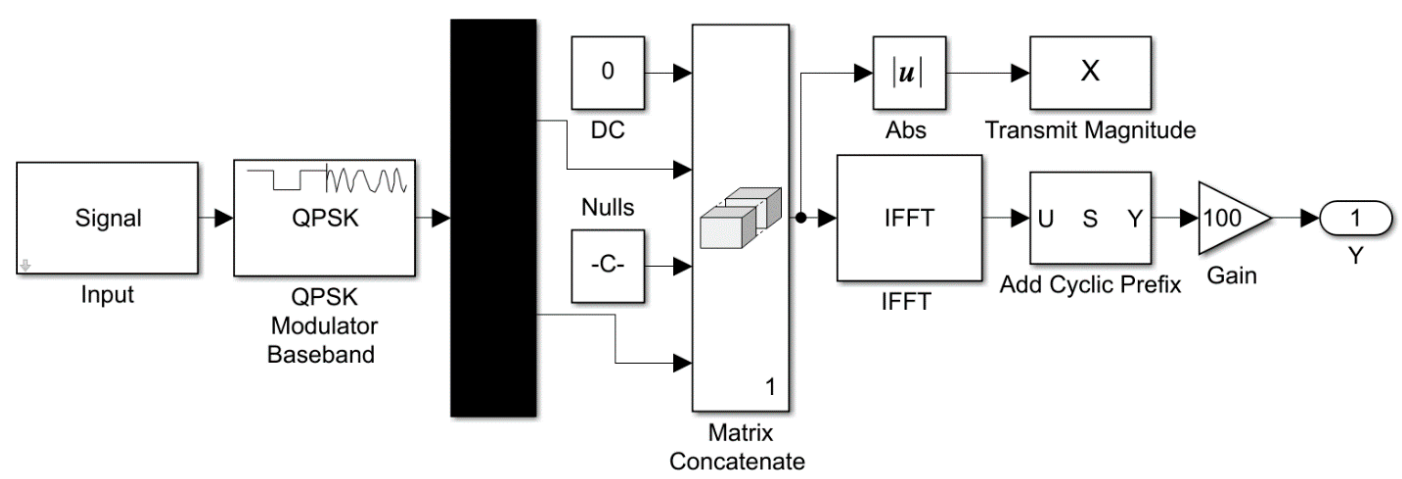

Figure 3. Transmitter operation for generating Orthogonal Frequency Division Multiplexing (OFDM) signal. 


\subsubsection{Receiver Process}

In the receiver process, as it appears in Figure 4, after down-sampling and ADC in the USRP receiver block, the first step is to remove the CP. Then FFT is carried out to transform the signal from the time domain into the frequency domain, and to frame the transformation to set the sampling mode of the output signal. The magnitude of the received signal is calculated in the frequency domain. Table 3 shows the testbed parameters utilized for the generation of the OFDM signal for both the transmitter and the receiver. These parameters are also pliable and can be adjusted according to different requirements and standards. Finally, features are extracted from WCSI to ensure accurate, efficient, or meaningful analysis. Further principal component analysis (PCA) is utilized to reduce the dimensionality and the classification of WCSI data by using different machine learning algorithms.

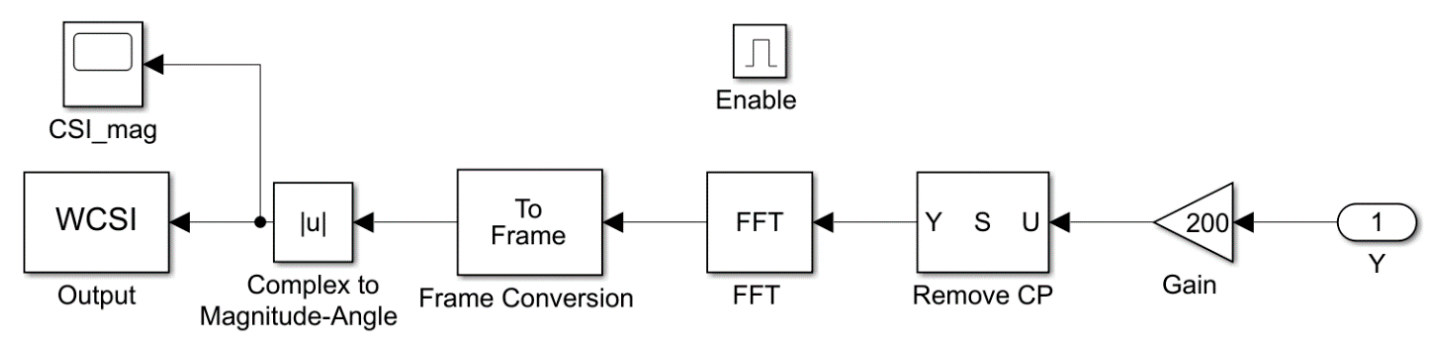

Figure 4. Receiver operation for extracting WCSI.

\subsection{Classification Approach}

The supervised machine learning algorithm (MLA) is used to develop a posture detection model that makes posture predictions based on WCSI data in the presence of uncertainty. As adaptive algorithms classify wrong and correct posture patterns in the WCSI data, when a learning computer is exposed to more experimental data, the computer improves its identification performance. We consider the entire set of WCSI data as a heterogeneous matrix. Rows of the matrix are 64-subcarriers of the OFDM signal for every single experiment, and columns of the matrix are features. We consider WCSI response data as a column vector where each row is labeled with the corresponding posture in the WCSI row data of whether the subject had wrong or correct posture. To train or fit a model, we choose an efficient algorithm that has more accuracy for a given set of WCSI data. Supervised MLA functionalities comprise an object and stream-lined framework. We can efficiently train different algorithms according to the data set. There are trade-offs among several characteristics of the algorithms, such as memory usage, speed of training, and accuracy achieved in the data. Once we validate the model, we can change it to enhance the accuracy. We can interactively train, validate, and tune MLA models. We can choose between different algorithms to train and validate models for binary or multi-class problems. After training various models, we compare their validation errors side-by-side, and then select the best model. Training a model with a validation approach protects against overfitting using cross-validation. Model accuracy is used as a diagnostic measures to reflect the validated model results and are measured as given in (1):

$$
\text { Accuracy }=\frac{\text { Number of correct predictions }}{\text { Total number of predictions }} \times 100 .
$$

We use binary classification, and accuracy can also be measured in terms of positives and negatives as given in (2):

$$
\text { Accuracy }=\frac{\mathrm{TP}+\mathrm{TN}}{\mathrm{TP}+\mathrm{TN}+\mathrm{FP}+\mathrm{FN}} \times 100
$$

where TP $=$ True Positives, $\mathrm{TN}=$ True Negatives, FP $=$ False Positives, and FN = False Negatives. 


\section{Experimental Setup}

The experiments were performed at Xidian University in the "Internet of Thing" Lab, China. The experimental setup for post-surgery activity detection is shown in Figure 5. Four males and one female of ages between 25 to 35 having different body structures, heights, and weights are given in Table 4 to perform the correct and wrong posture activity. The correct and wrong posture of the weight lifting activity literature and videos are used to train the participants [32]. Every participant performed both correct and wrong posture activities. Each activity was performed ten times to train the model more accurately and precisely. Figure $6 \mathrm{a}, \mathrm{b}$ shows the wrong and correct posture position of the spinal cord patient for the weight lifting activity as prescribed in [32]. All participants performed both activities in the normal routine of the laboratory with all the environmental effects, e.g., reflection, scattering, diffraction, shadowing, etc., to perform experiments. It is widely accepted that an empty environment contained less noise and interference. We considered an experimental setup with tables, chairs, cupboard desktop computers, and a crowded environment that has a large number of multi-paths effects to validate the effectiveness of the system in the process of building the pattern of the WCSI data. This experimental setup can be deployed in any real environment.

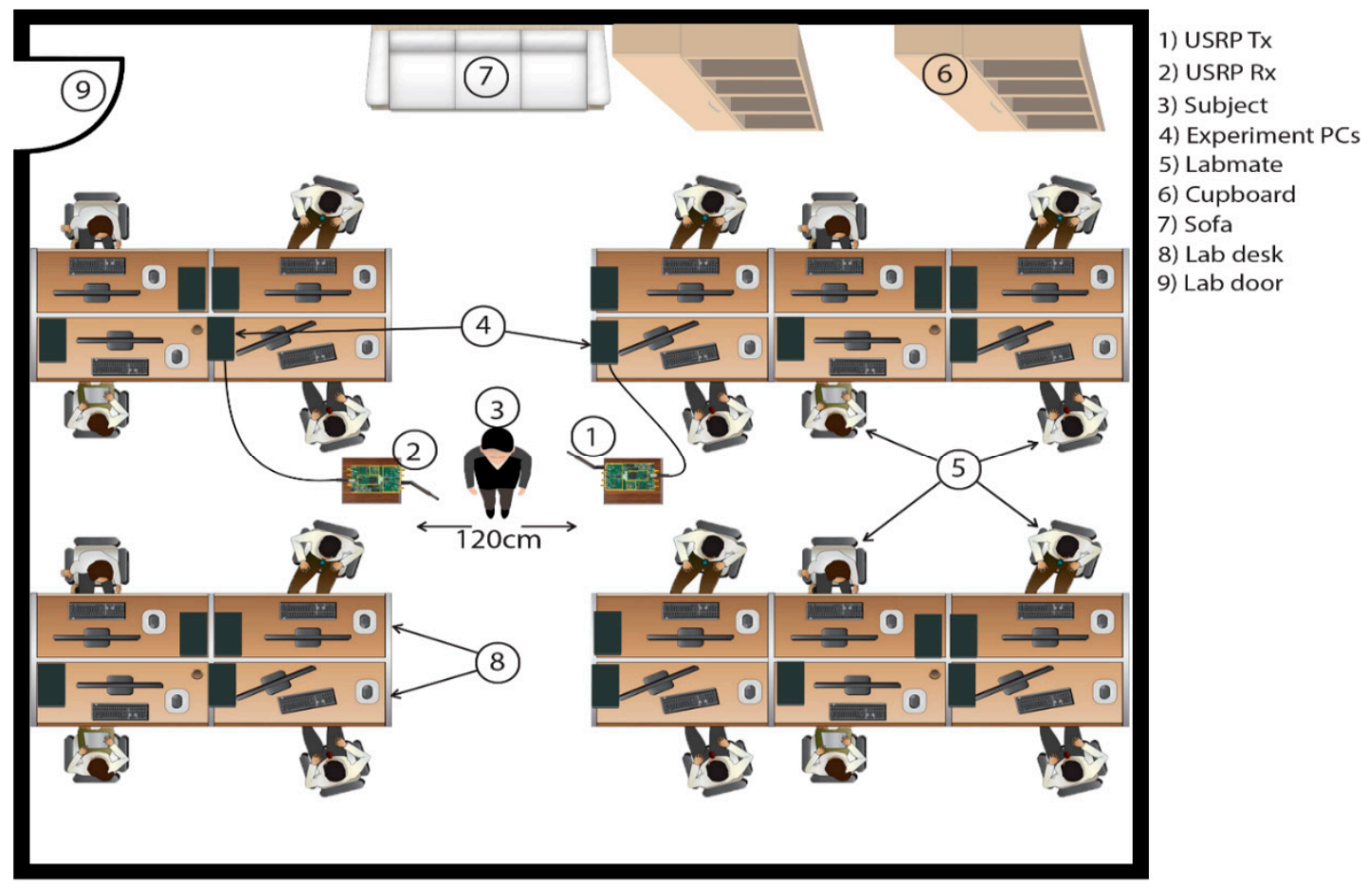

Figure 5. Experimental setup and lab environment.

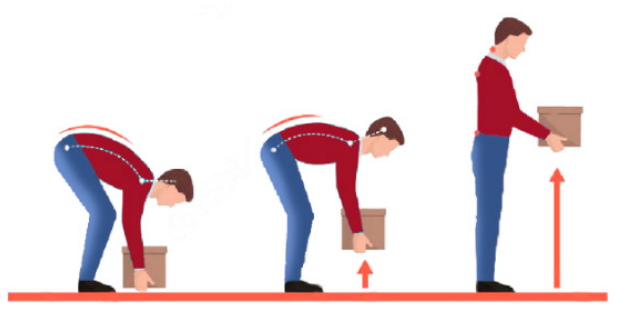

(a)

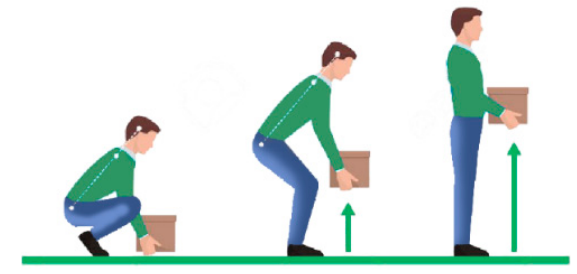

(b)

Figure 6. Expert prescribed posture: (a) wrong posture and (b) correct posture. 
Table 4. Subject participation in experiments.

\begin{tabular}{ccccc}
\hline Sr.No & Subject & Body structure & Height $(\mathbf{c m})$ & Weight $\mathbf{K g})$ \\
\hline 1 & Male & Ectomorph & 168 & 55 \\
2 & Male & Endomorph & 180 & 95 \\
3 & Female & Mesomorph & 168 & 60 \\
4 & Male & Mesomorph & 174 & 76 \\
5 & Male & Ectomorph & 176 & 60 \\
\hline
\end{tabular}

\section{Results and Discussion}

Results achieved and the performance of the testbed are evaluated in the following manner.

I. Correct and wrong activity magnitude response in the frequency domain.

II. Accuracy of the machine-learning algorithms model.

\subsection{Correct and Wrong Activity Magnitude Response in the Frequency Domain}

Extensive experiments were performed to design the testbed with settings for the values of different parameters for optimum and reliable results to ensure the testbed will work properly for real-world problems. While considering the initial testing of the system, we considered binary but multi motion activity to test the system. The results achieved for correct and wrong posture are shown in Figures 7 and 8 for all the 64 subcarriers. The magnitude response in the lab with other activities was going on, but the prominent amplitude changes were observed of the subject performing the activity. Although, the small-amplitude variation of the other students working in the lab are also observed because the system is very sensitive to human body motions. It is also observed that it gave strong amplitude changes in the LOS subject as compared to NLOS. NLOS motion is also present in the WCSI, but the response of LOS is more prominent. The randomly selected 10th subcarrier of both activities is shown to analyze the trend in Figure 9. Thus, this trend of WCSI can be useful in the classification of multi activities with multi motions. The achieved results show that both activities can easily be recognized by the naked eye by comparing both of the activities. Amplitude response in the frequency domain of the WCSI is prominent enough to apply supervised machine learning for the classification and justification of system monitoring capabilities.

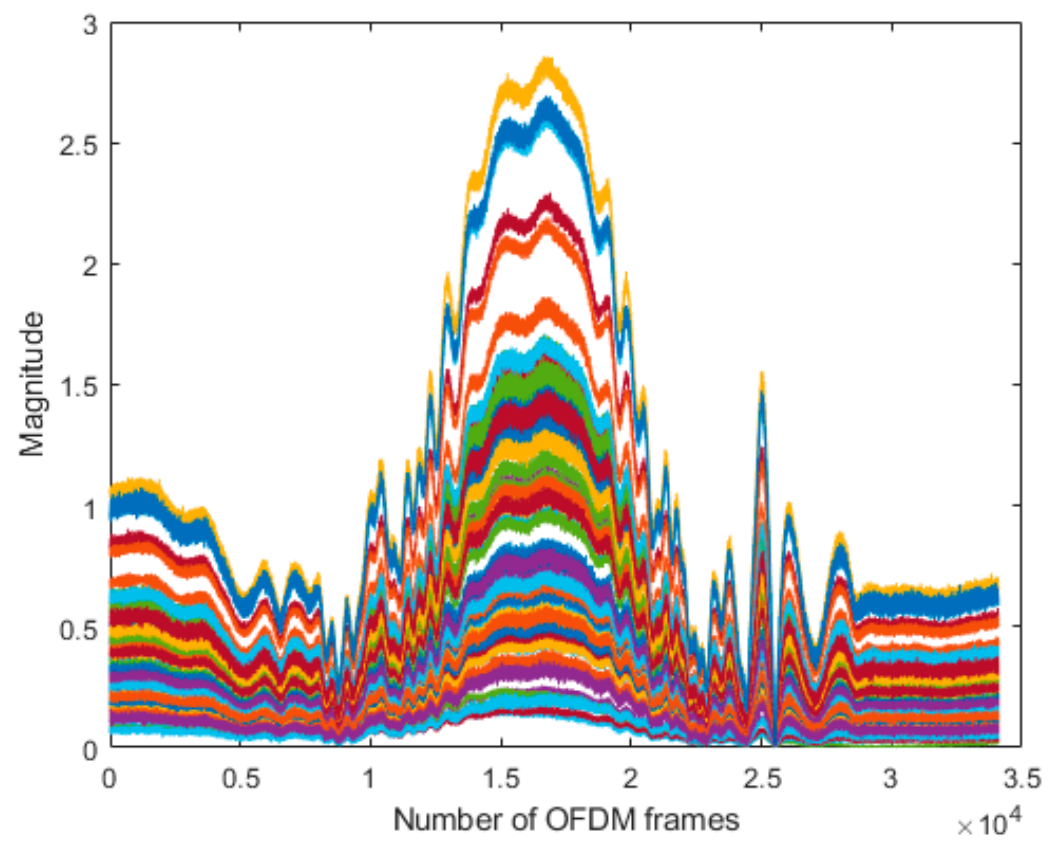

Figure 7. Result of correct activity using 64-subcarriers. 


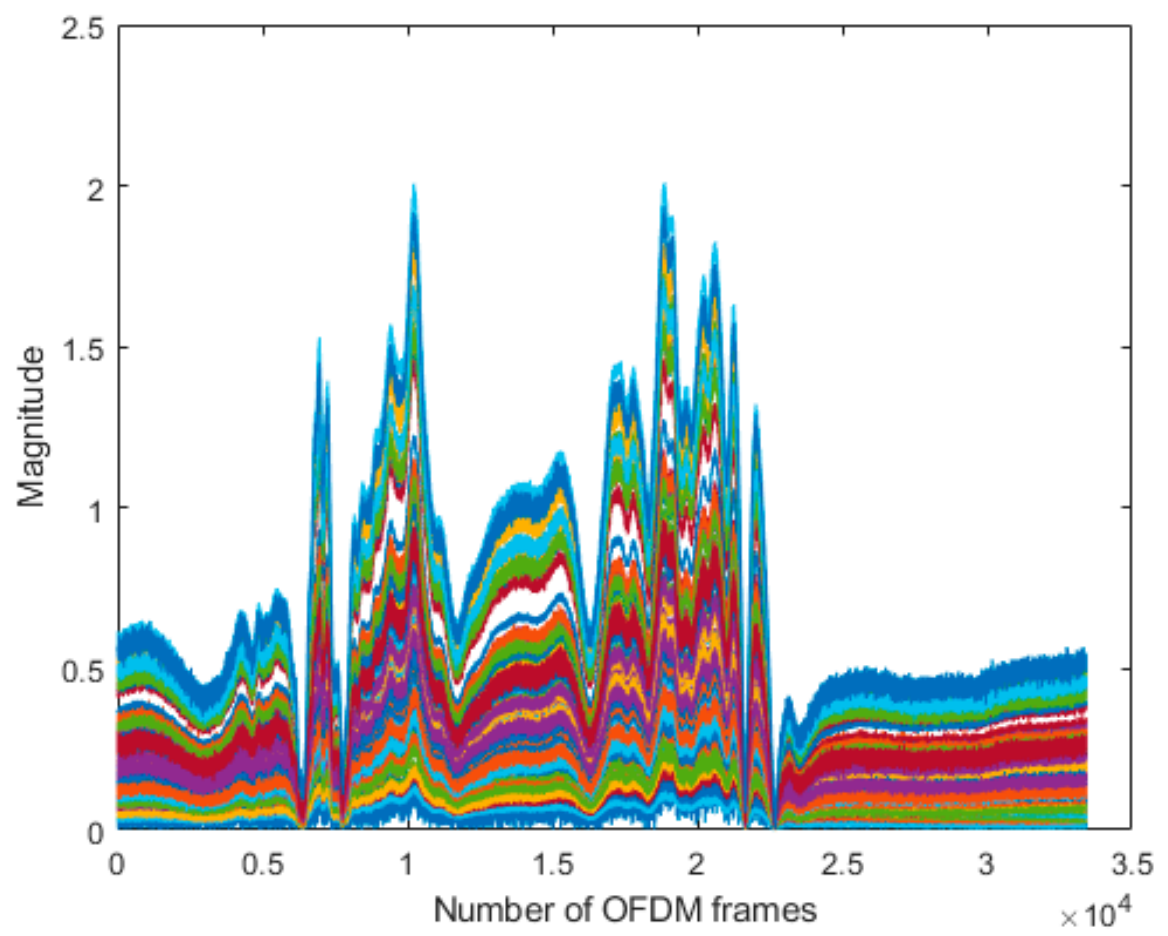

Figure 8. Result of wrong activity using 64-subcarriers.

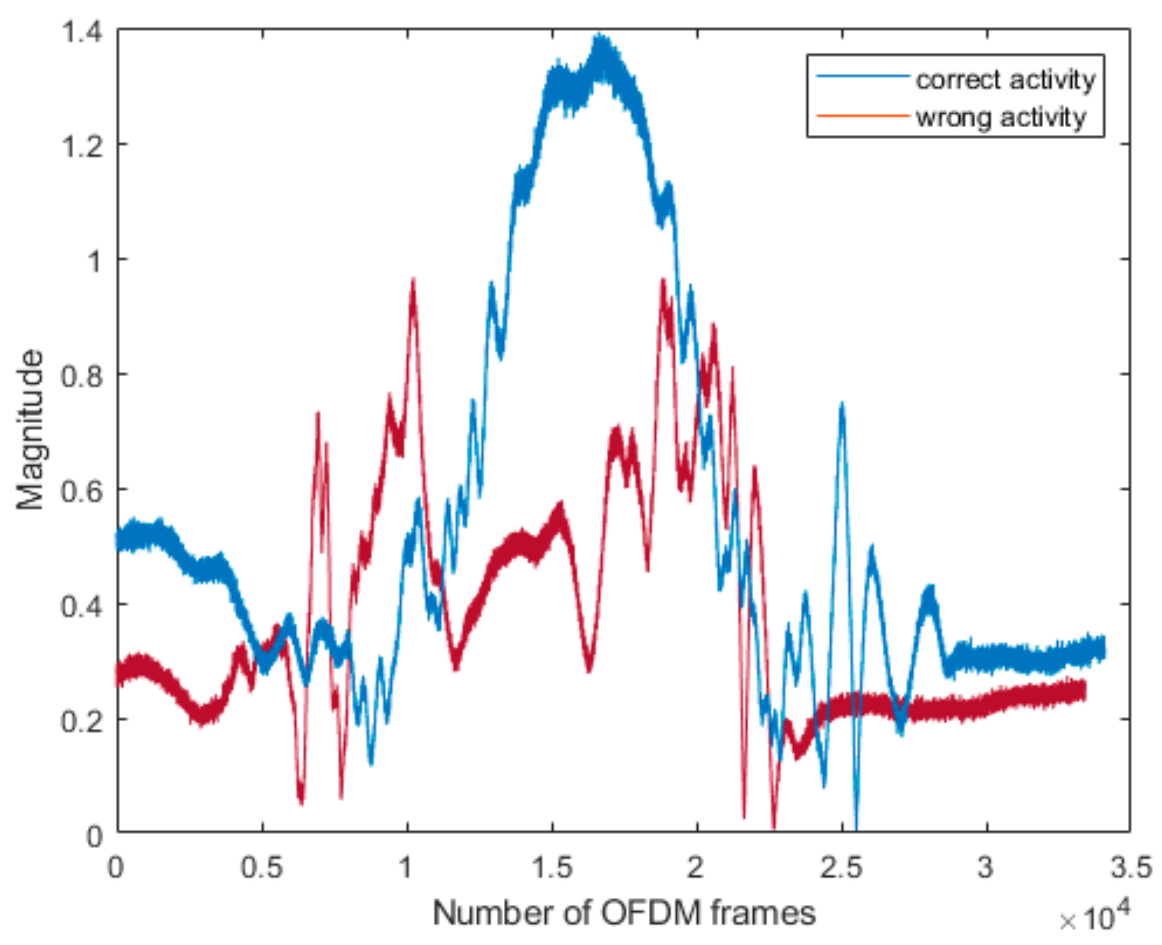

Figure 9. The comparison between the correct and wrong activities.

\subsection{Accuracy of the Machine Learning Algorithms Model}

We applied $90 \%$ of the WCSI data to train a model that classifies wrong and correct posture and $10 \%$ of the WCSI data to validate the trained model. We used five-fold cross-validation, which protects against overfitting. The following five well-known algorithms were selected to train the extracted features.

- $\quad$ Fine decision tree (FDT) 
- Linear discriminant analysis (LDA)

- Linear support vector machine (LSVM)

- $\quad$ Fine k-nearest neighbor (FKNN)

- Ensemble boosted trees (EBT)

Chart 1 shows the performance of each algorithm applied. The performance of fine KNN is best among all the five algorithms applied and can classify $99.6 \%$ of the post-surgery posture. The accuracy of the classifier is high due to binary classification. The result shows that the trained model is not over-fit to the training data set. The accuracy measurement depends on the operating system and can vary slightly. To confirm the accuracy of the model, it is necessary to place your device in the same way as described for collecting the training data. On the basis of the results achieved, we conclude that a non-contact sensing testbed can be implemented for post-surgery monitoring by exploiting artificial-intelligence techniques. This research can be carried out for multiclass, more complex post-surgery monitoring by using this testbed.

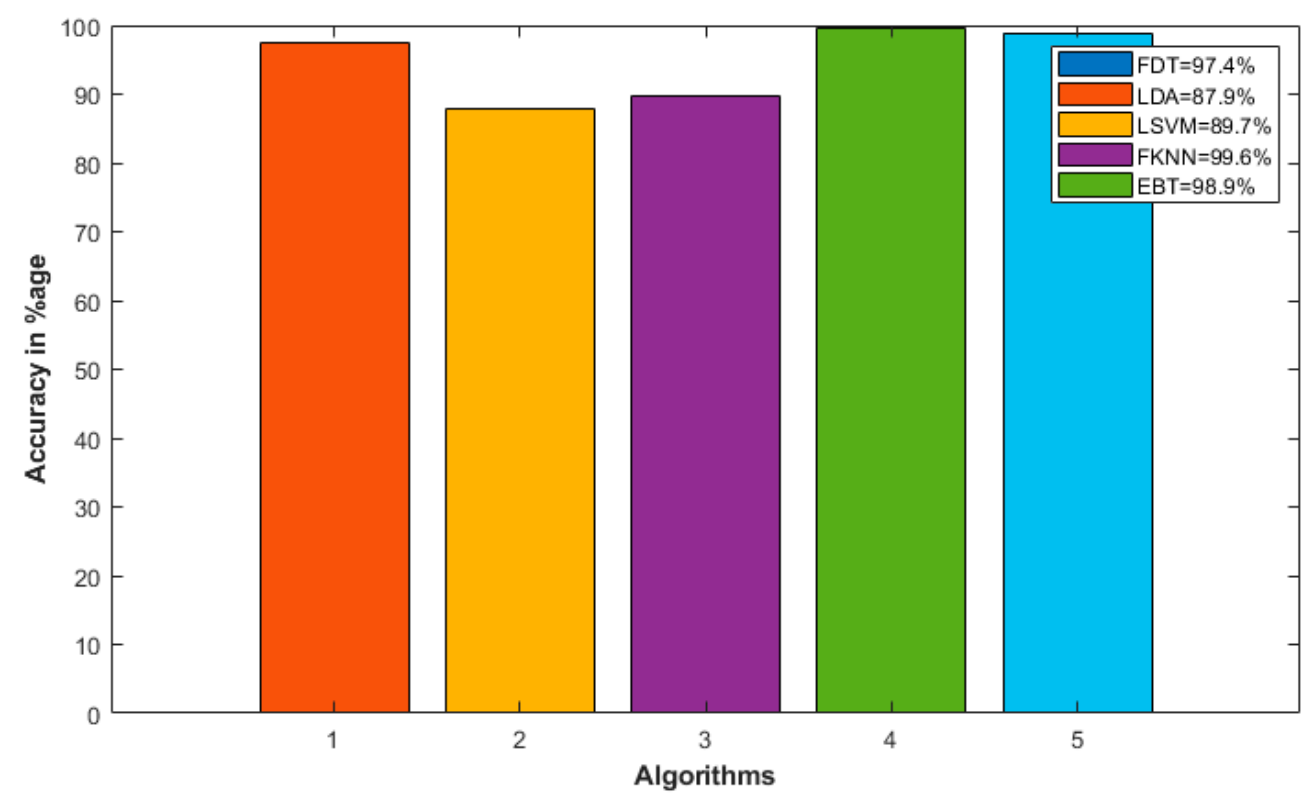

Chart 1. Accuracy of machine learning algorithms model.

\section{Conclusions and Future Recommendations}

In this paper, we designed a non-contact sensing testbed by exploiting artificial intelligence to classify post-surgery activities. In this research, we considered the correct posture of the spinal cord patients to lift the weight and classified the wrong posture to avoid any damage. We achieved $99.6 \%$ accuracy by applying the FKNN machine learning algorithm with PCA. Having adequate accuracy, this model can be used for real-world problems by reducing the cost, data moving, communication, and management.

This testbed can be utilized in the future for post-surgery monitoring of heart, eye, C-sections, and many healthcare applications. It can be further enhanced and explored in various fields. It can be deployed in earthquakes, wars, and emergency conditions to diagnose and monitor health. This system can be improved to mitigate channel noise, optimal gain, and long-distance detection. Still, there is a lot of research opening up by using this testbed to monitor multi-subjects and multi-activities simultaneously.

Author Contributions: Conceptualization, M.A.M.A.-h.; methodology, M.A.M.A.-h. and M.B.K.; software, M.A.M.A.-h. and M.B.K.; validation, M.B.K. and F.A.-T.; formal analysis, M.A.M.A.-h., M.B.K. and F.A.-T.; investigation, F.A.-T.; resources, X.Y.; data curation, F.A.-T. and N.Z.; writing-original draft preparation, 
M.A.M.A.-h. and M.B.K.; writing-review and editing, F.A.-T. and N.Z.; visualization, N.Z. and X.Y.; supervision, X.Y.; project administration, X.Y.; funding acquisition, X.Y. All authors have read and agreed to the published version of the manuscript.

Funding: National Natural Science Foundation of China 61301175.

Conflicts of Interest: The authors declare no conflict of interest.

\section{References}

1. Rahmani, A.M.; Gia, T.N.; Negash, B.; Anzanpour, A.; Azimi, I.; Jiang, M.; Liljeberg, P.J.F.G.C.S. Exploiting smart e-Health gateways at the edge of healthcare Internet-of-Things: A fog computing approach. Future Gener. Comput. Syst. 2018, 78, 641-658. [CrossRef]

2. Oueida, S.; Kotb, Y.; Aloqaily, M.; Jararweh, Y.; Baker, T.J.S. An edge computing based smart healthcare framework for resource management. Sensors 2018, 18, 4307. [CrossRef] [PubMed]

3. Al-Turjman, F.; Zahmatkesh, H.; Mostarda, L.J.I.A. Quantifying uncertainty in internet of medical things and big-data services using intelligence and deep learning. IEEE Access 2019, 7, 115749-115759. [CrossRef]

4. Deebak, B.; Al-Turjman, F.; Aloqaily, M.; Alfandi, O.J.I.A. An Authentic-Based Privacy Preservation Protocol for Smart e-Healthcare Systems in IoT. IEEE Access 2019, 7, 135632-135649. [CrossRef]

5. Samani, H.; Zhu, R.J.I.A. Robotic automated external defibrillator ambulance for emergency medical service in smart cities. IEEE Access 2016, 4, 268-283. [CrossRef]

6. Bickenbach, J.E.; Biering-Sorensen, F.; Knott, J.; Shakespeare, T.; Stucki, G.; Tharion, G. Understanding spinal cord injury. In International Perspectives on Spinal Cord Injury; Bickenbach, J.E., Ed.; WHO: Geneva, Switzerland, 2013; pp. 15-17.

7. Fleury, A.; Vacher, M.; Noury, N. SVM-based multimodal classification of activities of daily living in health smart homes: Sensors, algorithms, and first experimental results. IEEE Trans. Inf. Technol. Biomed. 2010, 14, 274-283. [CrossRef]

8. Kasteren, T.L.; Englebienne, G.; Kröse, B.J. An activity monitoring system for elderly care using generative and discriminative models. J. Pers. Ubiquitous Comput. 2010, 14, 489-498. [CrossRef]

9. Khan, B.; Yang, X.; Ren, A.; Al-hababi, M.; Zhao, N.; Guan, L.; Fan, D.; Aziz Shah, S. Design of Software Defined Radios Based Test bed for Activity Recognition. IEEE Access 2019, 7, 31083-31088. [CrossRef]

10. Nee, R.V.; Prasad, R. OFDM for Wireless Multimedia Communications; Artech House, Inc.: Norwood, MA, USA, 2000.

11. Siamak, Y.; Narui, H.; Dayal, S.; Ermon, S.; Valaee, S. A survey on behavior recognition using wifi channel state information. IEEE Commun. Mag. 2017, 55, 98-104.

12. Wang, Y.; Jiang, X.; Cao, R.; Wang, X. Robust indoor human activity recognition using wireless signals. Sensors 2015, 15, 17195-17208. [CrossRef]

13. Wang, H.; Zhang, D.; Wang, Y.; Ma, J.; Wang, Y.; Li, S. RT-Fall: A Real-Time and Contactless Fall Detection System with Commodity WiFi Devices. IEEE Trans. Mobile Comput. 2017, 16, 511-526. [CrossRef]

14. Wang, X.; Yang, C.; Mao, S. TensorBeat: Tensor Decomposition for Monitoring Multiperson Breathing Beats with Commodity WiFi. ACM Trans. Intell. Syst. Technol. (TIST) 2017, 9, 1-27. [CrossRef]

15. Al-Turjman, F.; Nawaz, M.H.; Ulusar, U.D.J.C.C. Intelligence in the Internet of Medical Things era: A systematic review of current and future trends. Comput. Commun. 2019, 150, 644-660. [CrossRef]

16. Vallabh, P.; Malekian, R. Fall Detection Monitoring Systems: A Comprehensive Review. J. Ambient Intell. Hum. Comput. 2018, 9, 1809-1833. [CrossRef]

17. Lotfi, A.; Albawendi, S.; Powell, H.; Appiah, K.; Langensiepen, C. Supporting Independent Living for Older Adults; Employing a Visual Based Fall Detection Through Analysing the Motion and Shape of the Human Body. IEEE Access 2016, 6, 70272-70282. [CrossRef]

18. Zhang, Z.; Conly, C.; Anthitsos, V. A Survey on Vision-based Fall Detection. In Proceedings of the 8th ACM International Conference on Pervasive Technologies Related to Assistive Environments, Corfu, Greece, 1-3 July 2015; pp. 1-7.

19. De Miguel, K.; Brunete, A.; Hernando, M.; Gambao, E. Home camera-based fall detection system for the elderly. Sensors 2017, 17, 2864. [CrossRef] 
20. Balakrishnan, G.; Durand, F.; Guttag, J. Detecting pulse from head motions in video. In Proceedings of the IEEE Conference on Computer Vision and Pattern Recognition (CVPR), Portland, OR, USA, 25-27 June 2013; pp. 3430-3437.

21. Jain, H.; Chen, H. A Portable Fall Detection and Alerting System Based on k-NN Algorithm and Remote Medicine. China Commun. Mag. 2015, 12, 23-31. [CrossRef]

22. Sabatini, A.M.; Ligorio, G.; Mannini, A.; Genovese, V.; Pinna, L. Prior-to- and Post-Impact Fall Detection Using Inertial and Barometric Altimeter Measurements. IEEE Trans. Neural Syst. Rehabil. Eng. 2016, 24, 774-783. [CrossRef]

23. Zigel, Y.; Litvak, D.; Gannot, I. A Method for Automatic Fall Detection of Elderly People Using loor Vibrations and Sound-Proof of Concept on Human Mimicking Doll Falls. IEEE Trans. Biomed. Eng. 2009, 56, 2858-2867. [CrossRef]

24. Luque, R.; Casilari, E.; Morón, M.J.; Redondo, G. Comparison and Characterization of Android-Based Fall Detection Systems. Sensors 2014, 14, 18543-18574. [CrossRef]

25. Garripoli, C.; Mercuri, M.; Karsmakers, P.; Soh, P.J.; Crupi, G.; Vandenbosch, G.A.E.; Pace, C.; Leroux, P.; Schreurs, D. Embedded DSP-Based Telehealth Radar System for Remote In-Door Fall Detection. IEEE J. Biomed. Health Inform. 2015, 19, 92-101. [CrossRef] [PubMed]

26. Jokanovíc, B.; Amin, M. Fall Detection Using Deep Learning in Range-Doppler Radars. IEEE Trans. Aerosp. Electron. Syst. 2018, 54, 180-189. [CrossRef]

27. Sugano, M.; Kawazoe, T.; Ohta, Y.; Murata, M. Indoor Localization System using RSSI Measurement of Wireless Sensor Network based on ZigBee Standard. In Proceedings of the 2006 IFIP International Conference on Wireless and Optical Communications, Bangalore, India, 11-13 April 2006; pp. 1-6.

28. Vasisht, D.; Kumar, S.; Katabi, D. Decimeter-Level Localization with a Single WiFi Access Point. In Proceedings of the USENIX Symposium on Networked Systems Design and Implementation (NSDI), Santa Clara, CA, USA, 16-18 March 2016; pp. 165-178.

29. Ali, K.; Liu, A.X.; Wang, W.; Shahzad, M. Recognizing Keystrokes Using WiFi Devices. IEEE J. Sel. Areas Commun. 2017, 35, 1175-1190. [CrossRef]

30. Zhao, M.; Li, T.; Abu Alsheikh, M.; Tian, Y.; Zhao, H.; Torralba, A.; Katabi, D. Through-wall human pose estimation using radio signals. In Proceedings of the IEEE Conference on Computer Vision and Pattern Recognition (CVPR), Salt Lake City, UT, USA, 19-21 June 2018; pp. 7356-7365.

31. Zhang, Z.; Ishida, S.; Tagashira, S.; Fukuda, A. Danger-pose detection system using commodity Wi-Fi for bathroom monitoring. Sensors 2019, 19, 884. [CrossRef] [PubMed]

32. Available online: http://www.sasksurgery.ca/patient/spine.html (accessed on 2 July 2020). 